\title{
Visualizing Flow Fields Around Colloids
}

\author{
A new technique for measuring displacements of tiny particles in a fluid \\ reveals a previously unseen hydrodynamic flow pattern that could \\ provide intel on the interface's mechanical properties.
}

\author{
By Christopher Crockett
}

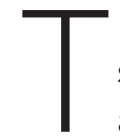

he thermal motions of tiny particles that sit on the surface of a fluid can induce flows. These flows are key to understanding a wide range of hydrodynamic phenomena, from the self-organization of particles on droplets to the properties of lipid membranes found in living cells. Now, researchers have come up with a way to visualize these flow fields for colloids that thermally jiggle on the surface of a film of water, allowing them to better understand the mechanical properties of this fluid interface [1].

A small particle on a fluid surface dances as fluid molecules bump into it. That dance then drives currents in the fluid far from the particle. In principle, researchers could see those currents by sprinkling other particles on the fluid and watching how they move. But there's a problem: those additional particles get thermally jostled by molecules too. With lots of independent thermal dances, it can be hard to reconstruct the fluid flows.
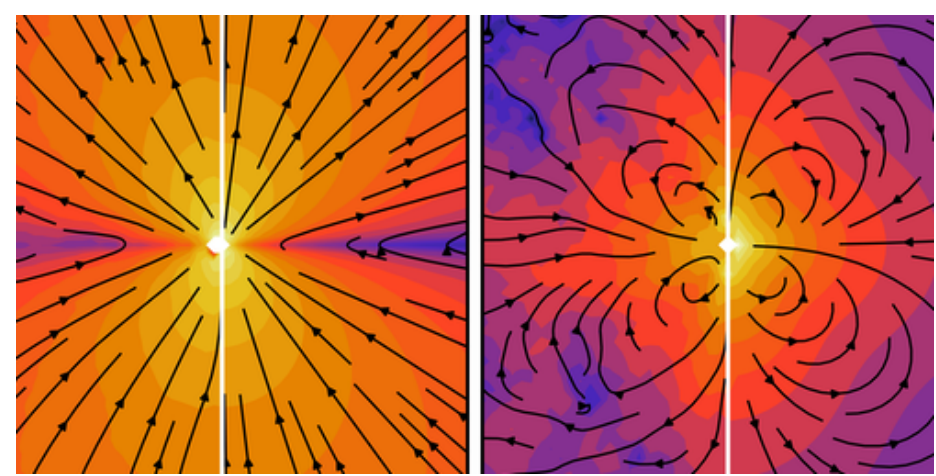

To get around this problem, Mehdi Molaei, Kathleen Stebe, and their colleagues at the University of Pennsylvania developed a technique that tracked the displacements of every particle in the fluid. They repeatedly analyzed video footage of micrometer-sized colloids at an air-water interface using a different colloid each time as a reference point. By calculating displacement correlations among roughly $10^{11}$ colloid pairs, the team reconstructed never-before-visualized hydrodynamic patterns swirling at the fluid interface.

These patterns reveal that the interface acts like a 2D incompressible sheet, a behavior the team attributed to trace amounts of contamination from surfactants-impurities that lower the surface tension of a fluid. While it was already known that surfactants can make a fluid's surface incompressible, the new experiments suggest that there is no way to remove this effect: The team found that even with a scant 1000 surfactant molecules per square micrometer-a concentration at which the surfactant is undetectable-the behavior persisted.

Christopher Crockett is a freelance writer based in Arlington, Virginia.

\section{REFERENCES}

1. M. Molaei et al., "Interfacial flow around Brownian colloids," Phys. Rev. Lett. 126, 228003 (2021).

Credit: M. Molaei et al. [1] 